\title{
UTILIZAÇÃO DO ESPECTRÔMETRO DE ABSORÇÃO ATÔMICA PARA DETERMINAÇÃO DE CLORIDRATO DE METFORMINA E CLORIDRATO DE RANITIDINA EM MEDICAMENTOS
}

\author{
Mariana Barbosa André de Souza ${ }^{\mathrm{a}}$, Klaiani Bez Fontana ${ }^{\mathrm{a}}$, Caroline Gonçalves ${ }^{\mathrm{b}, \#}$ e Eduardo Sidinei Chaves ${ }^{\mathrm{a}, *}$ \\ aDepartamento de Engenharia Química, Universidade Tecnológica Federal do Paraná, Câmpus Ponta Grossa, 84016-210 Ponta \\ Grossa - PR, Brasil

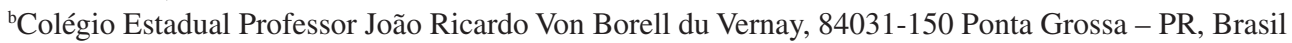

Recebido em 19/03/2017; aceito em 20/07/2017; publicado na web em 13/09/2017

\begin{abstract}
USE OF THE ATOMIC ABSORPTION SPECTROMETER FOR THE DETERMINATION OF METHPHORMINE AND RANITIDINE CHLORIDRATES IN MEDICINES. The utilization of atomic absorption spectrometer for metformin (MET) and ranitidine (RAN) chlorohydrates determination in medicines is proposed. The spectral lines of nickel and aluminum at 232.0 and $308.2 \mathrm{~nm}$ were selected for MET and RAN absorbance monitoring, respectively. The results were compared to those obtained by molecular absorption spectrometry at ultraviolet range (spectrophotometry) and condutometric titration. The figures of merit obtained were adequate for MET and RAN determination in medicines. The limit of detection (LOD) of $0.01 \mathrm{mg} \mathrm{L}^{-1}$ obtained for MET and RAN, were similar and even lowers than those obtained by spectrophotometry and comparable with those found in the literature. The precision evaluated by relative standard deviation (RSD) was lower than 6\%. The analytical application shows that in the analyzed samples the MET and RAN concentrations were in agreement with specified value in the label and the limits required by Brazilian legislation.
\end{abstract}

Keywords: atomic absorption spectrometer; molecular absorption; metformin; ranitidine; medicines.

\section{INTRODUÇÃO}

A utilização de um espectrômetro de absorção atômica de baixa resolução para determinações via espectrometria de absorção molecular (MAS) foi proposta na década de 70 para determinação de não-metais, como o enxofre e os halogênios. Nesta técnica, a adição de reagentes específicos possibilita a vaporização dos analitos na forma de moléculas diatômicas, portanto, segundo a Lei de Lambert-Beer a absorvância pode ser correlacionada com a concentração do analito. Contudo, as aplicações da MAS utilizando esta instrumentação são bastante limitadas, devido à baixa resolução espectral, potenciais interferências e pouca disponibilidade de linhas espectrais. ${ }^{1,2} \mathrm{O}$ surgimento da espectrometria de absorção atômica de alta resolução com fonte contínua (HR-CS AAS) ampliou a capacidade de determinações via absorção molecular. Desta maneira, a MAS utilizando HR-CS AAS vem sendo aplicada com sucesso para a determinação de bromo, ${ }^{3}$ enxofre ${ }^{4}$ flúor ${ }^{5}$ e cloro ${ }^{6}$ em diferentes matrizes.

A espectrometria de absorção atômica pode ser utilizada para determinação indireta de compostos moleculares, tais como cloridrato de propranolol, ranitidina, bromexina e flunarizina após reações de complexação e/ou precipitação dos analitos ${ }^{7,8}$ Entretanto, as medições diretas de absorção molecular utilizando um espectrômetro de absorção atômica (espectrômetro de AA) são possíveis, uma vez que moléculas absorvem na forma de bandas em amplas faixas na região ultravioleta (UV) ou visível (Vis) do espectro eletromagnético. Desta forma, as linhas espectrais emitidas por lâmpadas de cátodo oco, próximas ou coincidentes com o comprimento de onda de máxima absorção do analito, podem ser selecionadas para medições de absorção molecular em um equipamento inicialmente desenvolvido para medidas de absorção atômica. ${ }^{9}$ Neste sentido, a utilização do espectrômetro de AA para determinação direta de compostos moleculares foi proposta por Gomes e colaboradores

*e-mail: eschaves@utfpr.edu.br

\#Curso Técnico em Química para determinação de cromato e dicromato em soluções aquosas, monitorando a absorvância de cada espécie em linhas espectrais emitidas por lâmpadas de $\mathrm{Al}(396,1 \mathrm{~nm})$ e V $(437,9 \mathrm{~nm}) .{ }^{9}$ Além destes, cafeína e cloridrato de propranolol foram determinados em bebidas e formulações farmacêuticas por absorção molecular, monitorando as linhas espectrais do Fe (271,9 nm) e do $\mathrm{Mg}(285,2 \mathrm{~nm})$, respectivamente. ${ }^{10}$ Assim, o espectrômetro de AA pode ser utilizado para monitoramento da concentração de cloridrato de metformina (Cloridrato de N,N-dimetilimidodicarbonimídico diamida) (MET) e cloridrato de ranitidina (Cloridrato de N'-[2-[[[5-[(dimetilamino) metil]-2-furanil]metil]tio]etil]-N-metil-2-nitro-1,1-etenodiamina) (RAN) em medicamentos.

O MET é um agente anti-hiperglicêmico para tratamento de pacientes com diabetes tipo II. ${ }^{11}$ Este medicamento auxilia na normalização dos níveis elevados de glucose no sangue, reduz a glicogênese hepática e aumenta a captação periférica da glicose. ${ }^{12}$ O RAN é um inibidor gástrico para secreção ácida, utilizado geralmente para o tratamento de úlceras. ${ }^{13}$ Assim, o monitoramento da concentração destes em medicamentos comercializados no país é muito importante. Geralmente, a determinação de MET e RAN em diferentes matrizes tem sido realizada por técnicas eletroanalíticas, espectrométricas e cromatográficas. ${ }^{11,12,14-16}$ Adicionalmente, a Farmacopeia Brasileira recomenda que as determinações de MET e RAN sejam realizadas por meio de titulação com nitrato de prata, espectrofotometria e cromatografia líquida de alta eficiência (HPLC). ${ }^{17}$ Entretanto, na literatura não há trabalhos reportando a determinação direta de MET e RAN utilizando um espectrômetro de AA.

Neste trabalho, a utilização de um espectrômetro de AA para determinação de MET e RAN em medicamentos foi proposta e os resultados obtidos comparados com os determinados por titulações condutimétricas e espectrofotometria. As determinações dos analitos utilizando o espectrômetro de AA foram realizadas monitorando linhas espectrais emitidas por lâmpadas de cátodo oco de níquel (Ni) e alumínio (Al). Finalmente, as concentrações de MET e RAN foram determinadas em medicamentos de diferentes marcas e os 
valores comparados com os informados nas respectivas bulas e com os limites estabelecidos pela legislação brasileira.

\section{PARTE EXPERIMENTAL}

\section{Instrumentação}

As determinações de MET e RAN foram realizadas em um espectrômetro de absorção atômica (Perkin Elmer, AAnalyst 700, Alemanha) utilizando lâmpadas de cátodo oco de Ni e Al. Para MET e RAN, as absorvâncias foram monitoradas nas linhas espectrais de 232,0 nm (Ni) e 308,2 nm (Al), respectivamente. Além disso, as determinações foram realizadas por espectrofotometria, utilizando um espectrofotômetro de absorção molecular (FEMTO, 800XI Brasil) e uma cubeta de quartzo de $1,0 \mathrm{~cm}$. As titulações condutimétricas foram realizadas utilizando-se uma bureta (Digitrate Pro®, Jencons, Inglaterra) e uma célula de vidro encamisada, o controle de temperatura foi realizado com banho termostatizado (Solab, SL-152, Brasil) e as condutâncias obtidas com condutivímetro digital (Homis, 993, Brasil). Para facilitar a solubilização das amostras foi utilizado um banho ultrassônico de $40 \mathrm{kHz}$ e 135 Watts (Unique, USC1400A, Brasil) operando a temperatura ambiente $\left(25 \pm 3^{\circ} \mathrm{C}\right)$.

\section{Reagentes, soluções e amostras}

As soluções foram preparadas com água ultrapura (resistividade $18 \mathrm{M} \Omega \mathrm{cm}$ ) obtida de um sistema GEHAKA Master all 2000. Foram utilizados padrões de MET (NUTRIFARM, Brasil) e RAN (FRAGON, Brasil) com 99\% de pureza. Nitrato de prata (Nuclear, Brasil) foi utilizado nas titulações condutimétricas de MET e RAN nas amostras de medicamentos. Cloreto de sódio com 99\% de pureza (Neon, Brasil) foi utilizado na padronização da solução de nitrato de prata, utilizada nas titulações condutimétricas.

Foram analisadas 12 amostras de medicamentos, sendo 9 de MET (contendo 500, 850 e $1000 \mathrm{mg}$ de cloridrato de metformina por comprimido) e 3 amostras de RAN (com 150 e 300 mg de cloridrato de ranitidina por comprimido) de diferentes marcas adquiridas no comércio local da cidade de Ponta Grossa, Paraná. Considerando que as concentrações de MET e RAN são expressas em termos de mg do princípio ativo por comprimido, a massa média dos comprimidos para cada uma das marcas analisadas foi obtida por meio da pesagem de, pelo menos, 10 comprimidos. Neste caso, as massas médias obtidas para os comprimidos dos medicamentos analisados foram entre 0,53 - 1,04 g para MET e 0,27 - 0,73 g para RAN.

\section{Medidas usando espectrômetro de absorção atômica}

Os espectros de absorção molecular de MET e RAN foram previamente obtidos por espectrofotometria, a partir dos quais os comprimentos de onda de máxima absorção foram definidos, sendo estes 232,0 nm para MET e de 306,0 nm para RAN. Posteriormente, as linhas de emissão de lâmpadas de cátodo de Ni $(232,0 \mathrm{~nm})$ e Al (308,2 nm) foram selecionadas para medidas de absorvância, sendo estas as mais próximas possíveis dos comprimentos de onda de máxima absorção do MET e RAN. As determinações no espectrômetro de AA foram realizadas com o sistema de correção de fundo desligado, evitando assim, interferência durante as medições de absorvância.

As medidas utilizando um espectrômetro de AA foram realizadas posicionando uma cubeta de quartzo de $1 \mathrm{~cm}$ sobre o queimador do espectrômetro, na ausência de chama, de maneira que o feixe de radiação, emitido pela lâmpada de cátodo oco, incidisse na cubeta contendo a solução da amostra ou do padrão do analito, conforme descrito por Ferreira e colaboradores..$^{10}$ As medidas de absorvância foram realizadas com o próprio software e sistema óptico do equipamento, sem qualquer modificação.

\section{Procedimento}

Uma amostra contendo pelo menos 10 comprimidos de cada marca foi macerada em gral de porcelana. Posteriormente, alíquotas de $0,1 \mathrm{~g}$ foram pesadas em triplicata, diluídas em $100 \mathrm{~mL}$ de água, sonicadas em banho ultrassônico por 30 minutos, filtradas e posteriormente as concentrações de MET e RAN determinadas.

As determinações de MET por espectrofotometria foram realizadas no comprimento de onda de 232,0 nm e para as determinações utilizando espectrômetro de AA, as absorvâncias foram monitoradas na linha espectral do $\mathrm{Ni}(232,0 \mathrm{~nm})$. As curvas de calibração foram obtidas com padrões de MET com concentrações entre 1 e $30 \mathrm{mg} \mathrm{L}^{-1}$ diluídos em água ultrapura. As concentrações de MET nas amostras foram determinadas e expressas em termos de $\mathrm{mg}$ do princípio ativo/ comprimido. Na titulação condutimétrica, uma alíquota de $10 \mathrm{~mL}$ da solução filtrada foi avolumada para $200 \mathrm{~mL}$ e titulada com a solução de $\mathrm{AgNO}_{3}$ previamente padronizada $\left(0,01008 \mathrm{~mol} \mathrm{~L}^{-1}\right)$. As curvas de titulação de condutância corrigida versus volume de titulante foram obtidas, o ponto final da titulação foi determinado e, juntamente com a estequiometria da reação envolvida, utilizados para o cálculo de concentração de MET nos comprimidos.

As determinações de RAN foram realizadas de forma similar as determinações de MET. As leituras foram realizadas por espectrofotometria no comprimento de onda de $306,0 \mathrm{~nm}$ e no espectrômetro de AA, as absorvâncias foram medidas na linha espectral do Al em 308,2 nm, por ser a mais próxima do comprimento de máxima absorção do RAN (306,0 nm). As curvas de calibração foram obtidas com padrões de RAN com concentrações entre 1 e $40 \mathrm{mg} \mathrm{L}^{-1}$ diluídos em água ultrapura. As concentrações de RAN nas amostras foram determinadas e expressas em termos de $\mathrm{mg}$ do princípio ativo/ comprimido. Na titulação condutimétrica, uma alíquota de $20 \mathrm{~mL}$ da solução filtrada foi avolumada para $200 \mathrm{~mL}$ e titulada com a solução de $\mathrm{AgNO}_{3}$ previamente padronizada $\left(0,01008 \mathrm{~mol} \mathrm{~L}^{-1}\right)$. As curvas de titulação de condutância corrigida versus volume de titulante foram obtidas, o ponto final da titulação foi determinado e, juntamente com a estequiometria da reação envolvida, utilizados para o cálculo de concentração de RAN nos comprimidos.

\section{RESULTADOS E DISCUSSÃO}

\section{Figuras de mérito}

Na Figura 1 estão apresentadas as curvas de calibração para MET e RAN obtidas com o espectrômetro de AA e por espectrofotometria. Para MET, a faixa linear de calibração obtida por ambas as técnicas foi de 1 a $30 \mathrm{mg} \mathrm{L}^{-1}$. Entretanto, para RAN a faixa linear de calibração obtida por espectrofotometria foi de 1 a $20 \mathrm{mg} \mathrm{L}^{-1} \mathrm{e}$, utilizando o espectrômetro de AA, esta foi ampliada até $40 \mathrm{mg} \mathrm{L}^{-1}$. Neste caso, a faixa de calibração obtida para RAN foi relativamente superior à encontrada por Khalil e colaboradores ${ }^{7}$ para determinação indireta de cloridratos de ranitidina, bromexina e flunarizina em formulações farmacêuticas utilizando espectrometria de absorção atômica após precipitação com $\left[\mathrm{Cd}(\mathrm{SCN})_{4}\right]_{2}$ e / ou $\left[\mathrm{Co}\left(\mathrm{NO}_{2}\right)_{6}\right]_{3}$. Segundo os autores, RAN pode ser determinada na faixa de concentração de 1,26 a $12,61 \mathrm{mg} \mathrm{L}^{-1}$.

Comparando com a espectrofotometria, a ampliação da faixa linear de calibração em determinações moleculares usando o espectrômetro de AA foi também observada na determinação de cafeína e cloridrato de propranolol, monitorando linhas espectrais de lâmpadas de cátodo oco de Fe e Mg, respectivamente. Segundo os autores, ${ }^{10}$ uma 
das vantagens da utilização do espectrômetro de AA para medidas de compostos moleculares é a ampliação da faixa linear de calibração para determinação de alguns compostos. Considerando que a radiação monocromática é um dos termos mais importantes para aplicação da lei de Lambert-Beer, a ampliação da faixa linear, neste caso, está relacionada à radiação específica emitida pela lâmpada de cátodo oco.

As figuras de mérito obtidas para determinação de MET e RAN no espectrômetro de AA e por espectrofotometria estão apresentadas na Tabela 1. Os coeficientes de correlação linear obtidos para as
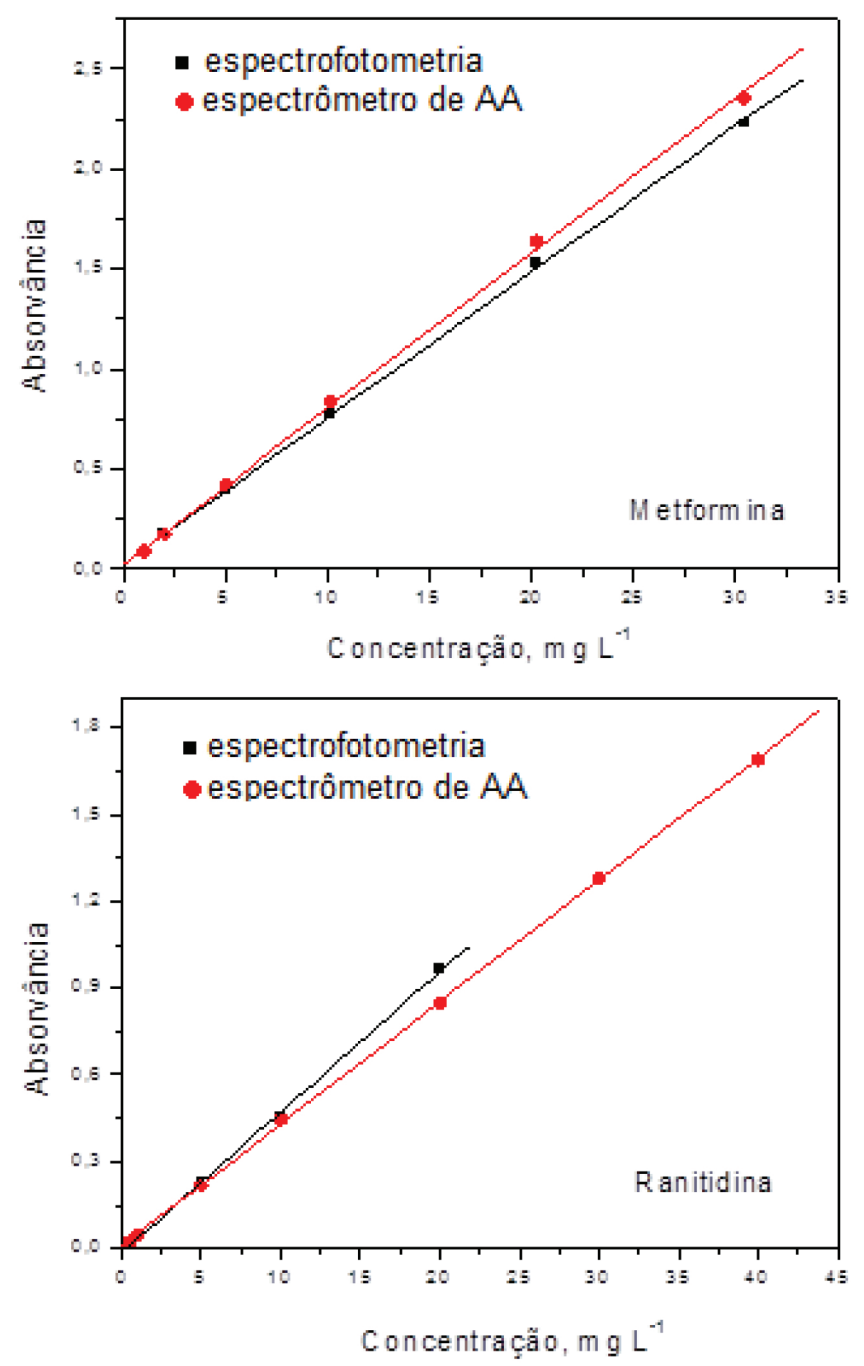

Figura 1. Curvas de calibração obtidas para cloridratos de metformina (MET) e ranitidina (RAN) utilizando espectrômetro de absorção atômica (espectrômetro de $A A$ ) e espectrofotometria determinações utilizando espectrômetro de AA foram satisfatórios, com valores de $\mathrm{R}^{2}>0,99$. As determinações realizadas com o espectrômetro de AA apresentaram sensibilidade, avaliadas pelos coeficientes de correlação angular das curvas de calibração, similares as obtidas por espectrofotometria. Os valores do limite de detecção (LOD) foram calculados considerando 3 vezes o desvio padrão de dez medidas consecutivas do branco das amostras dividido pelo valor da inclinação da respectiva curva de calibração e os limites de quantificação (LOQ) definidos como 3,3 vezes o LOD. Como observado, os valores de LOD foram inferiores aos obtidos por espectrofotometria. Considerando a concentração de MET e RAN normalmente encontradas em medicamentos, os LODs obtidos são adequados para determinação destes compostos em medicamentos. A determinação de RAN em medicamentos foi realizada por Malagutti e colaboradores ${ }^{16}$ utilizando-se ultramicroeletrodo de platina com eletrodepósito de mercúrio e o eletrodo de gota pendente de mercúrio. Com este método, o valor de LOD obtido para determinação potenciométrica de RAN foi de $0,011 \mathrm{mg} \mathrm{L}^{-1}$, sendo este valor similar ao LOD encontrado para determinação de RAN e MET utilizando espectrômetro de AA.

\section{Verificação da exatidão e precisão}

A verificação da exatidão das determinações de MET e RAN com o espectrômetro de AA foi avaliada por meio da comparação dos resultados obtidos por espectrofotometria e titulação condutimétrica, utilizando nitrato de prata como titulante. Na Tabela 2 estão apresentados os resultados obtidos para as determinações de MET e RAN em amostras de medicamentos, utilizando as diferentes técnicas analíticas.

O teste de Tukey foi aplicado e os resultados sugerem que, para um nível de significância de 5\%, não há diferença significativa entre os resultados obtidos para MET no espectrômetro de AA, espectrofotometria e titulação condutimétrica, demostrando uma boa concordância entre resultados obtidos no espectrômetro de AA e as demais técnicas. Para amostra contendo RAN, os resultados obtidos no espectrômetro de AA e espectrofotometria não apresentaram diferença significativa entre as concentrações obtidas. Entretanto, a concentração de RAN obtida por titulação condutimétrica foi levemente superior à concentração obtida com o espectrômetro de AA e por espectrofotometria, sendo considerado estatisticamente diferente. Contudo, se considerarmos o desvio padrão, podemos observar que há uma concordância satisfatória entre os resultados obtidos para RAN utilizando as diferentes técnicas. A precisão das medidas realizadas no espectrômetro de AA, avaliada por meio do desvio padrão relativo (RSD), apresentou valores de RSD $(n=3)<6 \%$, indicando a boa precisão das medidas. Além disso, a precisão obtida para determinações utilizando o espectrômetro de AA para determinação de MET e RAN foi similar à obtida para determinação destes analitos em amostras de medicamentos por titulação condutimétrica e por potenciometria. ${ }^{12,16}$

Tabela 1. Figuras de mérito para determinação de MET e RAN por espectrômetro de AA e espectrofotometria

\begin{tabular}{|c|c|c|c|c|}
\hline \multicolumn{5}{|c|}{ Cloridrato de Metformina (MET) } \\
\hline & Equação da reta & $\mathrm{R}^{2}$ & $\operatorname{LOD}\left(\mathrm{mg} \mathrm{L}^{-1}\right)$ & LOQ $\left(\mathrm{mg} \mathrm{L}^{-1}\right)$ \\
\hline Espectrômetro de AA & $y=0,0741 x+0,0306$ & 0,9986 & 0,01 & 0,03 \\
\hline Espectrofotometria & $\mathrm{y}=0,0709 \mathrm{x}+0,0434$ & 0,9938 & 0,04 & 0,13 \\
\hline \multicolumn{5}{|c|}{ Cloridrato de Ranitidina (RAN) } \\
\hline & Equação da reta & $\mathrm{R}^{2}$ & $\operatorname{LOD}\left(\mathrm{mg} \mathrm{L}^{-1}\right)$ & LOQ $\left(\mathrm{mg} \mathrm{L}^{-1}\right)$ \\
\hline Espectrômetro de AA & $y=0,0422 x+0,0067$ & 0,9999 & 0,01 & 0,03 \\
\hline Espectrofotometria & $\mathrm{y}=0,0485 \mathrm{x}-0,0097$ & 0,9996 & 0,06 & 0,20 \\
\hline
\end{tabular}

* LOD e LOQ instrumentais. 
Tabela 2. Concentração de MET e RAN (expressas em mg do princípio ativo/comprimido) em diferentes amostras obtidas utilizando as técnicas espectrômetro de AA, espectrofotometria e titulação condutimétrica. Média \pm desvio padrão $(\mathrm{n}=3)$

\begin{tabular}{lcc}
\hline \multicolumn{3}{c}{ Cloridrato de Metformina (MET) } \\
\hline Amostra & Técnica & $\begin{array}{c}\text { Concentração } \\
(\mathrm{mg} / \text { comprimido })\end{array}$ \\
\hline \multirow{3}{*}{ MB } & Espectrômetro de AA & $494 \pm 2^{\mathrm{a}}$ \\
& Espectrofotometria & $509 \pm 19^{\mathrm{a}}$ \\
& Titulação condutimétrica & $480 \pm 7^{\mathrm{a}}$ \\
\hline \multirow{3}{*}{ MC } & Espectrômetro de AA & $503 \pm 6^{\mathrm{a}}$ \\
& Espectrofotometria & $491 \pm 14^{\mathrm{a}}$ \\
\hline \multirow{3}{*}{ Amostra } & Titulação condutimétrica & $483 \pm 8^{\mathrm{a}}$ \\
\hline \multirow{3}{*}{ RB } & Cloridrato de Ranitidina (RAN) \\
& Técnica & Concentração \\
& Espectrômetro de AA & $148 \pm 1^{\mathrm{a}}$ \\
& Espectrofotometria & $153 \pm 5^{\mathrm{a}}$ \\
\hline
\end{tabular}

*Concentrações acompanhadas com diferentes letras mostram diferença significativa, considerando um nível de significância de 5\%. Massas médias dos comprimidos: $\mathrm{MB}$ e MC: 0,95 g e RB: 0,73 g.

\section{Aplicação analítica}

O espectrômetro de AA foi utilizado para determinação de MET e RAN em amostras de medicamentos de diferentes marcas. Os resultados obtidos para as determinações estão apresentados na Figura 2 , juntamente com a representação dos valores declarados na bula dos medicamentos, em linhas sólidas, e a variação máxima aceitável pela legislação de $\pm 5 \%$ (MET) $\pm 10 \%$ (RAN) em relação ao valor especificado, ilustrada pelas linhas pontilhadas. Foram analisadas 9 amostras de comprimidos contendo MET em concentrações de 500, 850 e $1000 \mathrm{mg}$ por comprimido e os resultados estão apresentados na Figura 2A. Além disso, 3 amostras contendo RAN em concentrações de 150 e $300 \mathrm{mg}$ por comprimido também foram analisadas e os resultados obtidos são mostrados na Figura 2B.

Segundo a Farmacopeia Brasileira, comprimidos de MET devem conter no mínimo $95 \%$ e no máximo $105 \%$ da quantidade declarada na bula, enquanto comprimidos de RAN devem possuir no mínimo $90 \%$ e no máximo $110 \%$ da quantidade declarada. ${ }^{17}$ Todas as amostras analisadas apresentam valores concordantes de MET e RAN com as respectivas especificações das bulas dos medicamentos. De modo geral, as determinações realizadas no espectrômetro de AA resultaram em valores de concentração para MET e RAN com erros inferiores a $5 \%$ e $10 \%$, respectivamente, em relação ao valor de referência de cada medicamento. Sendo assim, todas as marcas analisadas estavam de acordo com os limites especificados pela farmacopeia brasileira. Sartori e colaboradores ${ }^{12}$ determinaram concentração de MET em três amostras de medicamentos contendo diferentes concentrações de MET por titulação condutimétrica. Os resultados obtidos mostraram boa concordância com as concentrações de MET informadas na bula de cada amostra. A RAN foi determinada em três diferentes marcas por Malagutti e colaboradores, ${ }^{16}$ os resultados obtidos pelos autores também mostraram boa concordância com os informados na bula do medicamento.

\section{CONCLUSÃO}

A utilização do espectrômetro de AA para determinação das

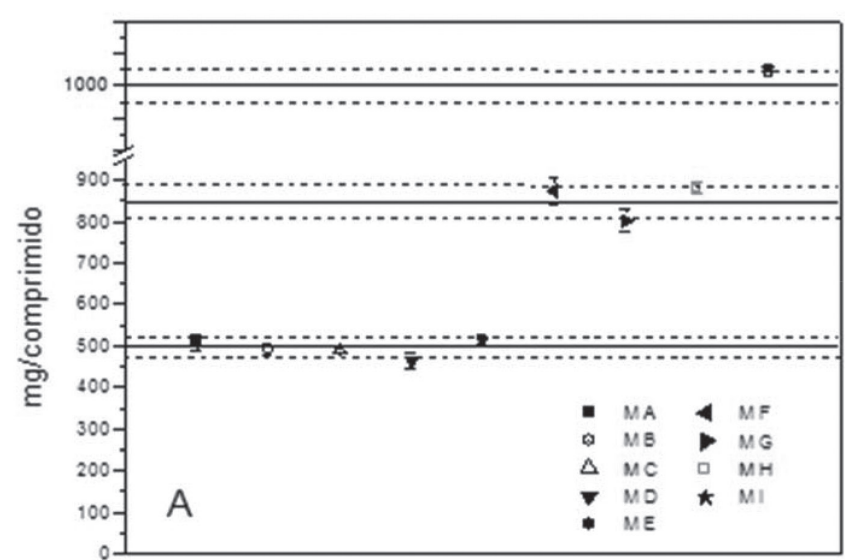

Cloridrato de Metformina

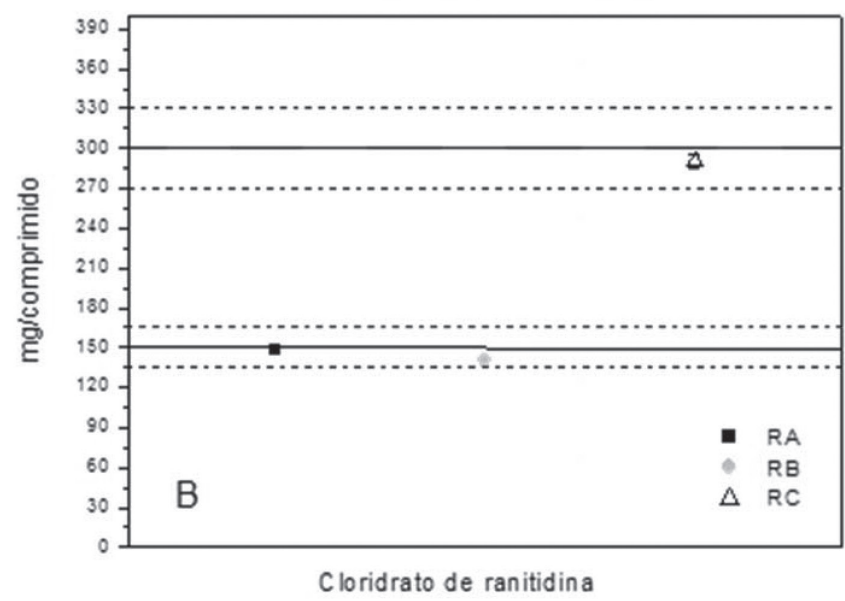

Figura 2. Concentrações de MET (A) e RAN (B), expressas em mg do princípio ativo por comprimido, obtidas no espectrômetro de AA para amostras comerciais de medicamentos, comparadas com as concentrações declaradas nas bulas (linha sólida) e as respectivas variações máximas de $\pm 5 \%$ para MET e $\pm 10 \%$ RAN (linha pontilhada) em relação à quantidade declarada. Amostras MA, MB, MC, MD, ME: 500 mg; MF, MG, MH 850 mg e MI 1000 mg de MET/comprimido. RA, RB:150 mg e RC: $300 \mathrm{mg}$ de RAN/comprimido

concentrações de MET e RAN em medicamentos foi realizada com sucesso. Os resultados obtidos para as concentrações de MET e RAN, utilizando espectrômetro de AA, apresentaram valores concordantes de concentração com os valores obtidos por titulações condutimétricas, utilizando nitrato de prata como titulante, e por espectrofotometria. Os medicamentos analisados apresentaram valores de concentração para MET e RAN concordantes com os valores especificados nas respectivas bulas e o estabelecido pela legislação brasileira.

\section{AGRADECIMENTOS}

À Universidade Tecnológica Federal do Paraná, CNPq e Fundação Araucária.

\section{REFERÊNCIAS}

1. Welz, B.; Sperling, M.; Atomic Absorption Spectrometry, $3^{\text {rd }}$ ed.; WileyVCH: Weinheim, 1999.

2. Butcher, D. J.; Anal. Chim. Acta 2013, 804, 1.

3. Flórez, M. R.; Resano, M.; Spectrochim. Acta, Part B 2013, 88, 32.

4. Mior, R.; Morés, S.; Welz, B.; Carasek, E.; Andrade, J. B.; Talanta 2013, 106, 368 . 
5. Ozbek, N.; Akman, S.; Spectrochim. Acta, Part B 2012, 69, 32.

6. Pereira, E. R.; Welz, B.; Lopez, A. H. D.; Gois, J. S.; Caramori, G. F.; Borges, D. L. G.; Carasek, E.; Andrade, J. B.; Spectrochim. Acta, Part $B$ 2014, 102, 1.

7. Khalil, S.; Ibrahim, S. A.; Zedan, F. I.; Abd-El-monem, M. S.; Chem. Anal. 2005, 50, 897.

8. El-Ries, M. A.; Abou Attia, F. M.; Ibrahim, S. A.; J. Pharm. Biomed. Anal. 2000, 24, 179.

9. Gomes, M. S.; Trevizan, L. C.; Nóbrega, J. A.; Gouveia, S. T.; Rocha, F. R. P.; Quim. Nova 2006, 29, 868.

10. Ferreira, B. L.; Vitali, L.; Chaves, E. S.; J. Braz. Chem. Soc. 2016, 27, 794.
11. Modi, D. K.; Patel, B. H.; J. Liq. Chromatogr. Relat. Technol. 2012, 35, 28.

12. Sartori, E. R.; Suarez, W. T.; Fatibello-Filho, O.; Quim. Nova 2009, 32, 1947.

13. Kiszkiel, I.; Starczewska, B.; Le'sniewska, B.; Pózniak, P.; J. Pharm. Biom. Anal. 2015, 106, 85.

14. Tian, X-J.; Song, J.-F.; J. Pharm. Biomed. Anal. 2007, 44, 1192.

15. Abdel-Ghany, M. F.; Abdel-Aziz, O.; Mohammed, Y. Y.; Spectrochim. Acta, Part A 2015, 149, 30.

16. Malagutti, A. R.; Mazo, L. H.; J. Braz. Chem. Soc. 2003, 14, 274.

17. Agência Nacional de Vigilância Sanitária; Farmacopeia Brasileira, v. 2, Brasília, 2010. 